\title{
Models in Fluid Dynamics
}

\author{
Michael Heidelberger
}

According to the covering law account of scientific explanation, a phenomenon is explained by "subsuming it under general laws, i.e. by showing that it occurred in accordance with these laws, in virtue of the realization of certain specified antecedent conditions." (Hempel 1965, 246) It has become apparent that this conception implies a "structural identity of explanation and prediction." (Ibid., 367) Prediction and explanation, this thesis says, differ from each other not in logical structure but only in the temporal relation between the moment when the phenomenon happens and the time when a statement about the subsumption is uttered. When an event is explained it has already happened, whereas when it is predicted its occurrence lies in the future. Everything else stays the same. Subsequent criticism has convincingly shown that this assertion of structural identity is deeply flawed and that the covering law account of explanation is responsible for this failure. If a barometric reading correctly predicts a storm, this reading cannot be taken to explain it, although it should do just that if the covering law account, along with its identity claim, were correct. The moral to be drawn is that explanation and prediction have to be distinguished.

It seems to me that there is another identity thesis that is closely related to the former and no less effective, although it is less frequently discussed. It can be called "structural identity of explanation and application" of a theory. It says that applying a theory to a special problem (an event or a specific empirical regularity) is a matter of deriving this case from the fundamental principles of the theory under suitable circumstances. To apply a theory to a problem means generating a description of the case in question and showing that it is implied by the principles. When the linear oscillator is explained by Newtonian mechanics, we produce a description of it and show how it can be derived from Newton's laws. The only difference between explanation and application lies in the way the antecedent conditions are given: When a theory is to be applied to a special problem, the problem itself delivers us the antecedent conditions whereas in an explanation of a problem some imagination is needed to come up with the appropriate circumstances for the case in question. I think that Nancy Cartwright raises the same issue when she rejects the widespread view that scientific theories "explain by dint of the description they give of reality," i.e. that "once the job of describing is done, science can shut down.” (Cartwright 1983, 44) 
In the following I would like to criticise the second identity thesis and contribute to a more adequate account of what it means to apply a theory to special problems. In particular, I would like to deal with the way a physical theory is applied to engineering problems. I will do so by concentrating on the example of modern fluid dynamics as a case study. It will become clear that modelling is central to this discipline and that different concept of explanation and application have been developed that take the role of models into account.

\section{Prandtl's concept of the boundary layer}

Fluid dynamics stands out from other engineering disciplines insofar as its modern history seems to be less "messy" and more straightforward than, for example, the history of electrical engineering. Its modern "applicational turn," as one might call it, was relatively abrupt and occurred in a special and isolated local setting. The onset of modern model building in fluid dynamics can easily be dated. It came at the beginning of the 20th century with the work of Ludwig Prandtl (1875-1953), who found a way to bring together the purely empirical engineering tradition of hydraulics and the purely theoretical mathematical tradition of rational mechanics as it had developed in the 18th century. (For Prandtl see Meier 2000, Morrison 1999, Siekmann 1998, Gad-el-Hak 1998, Anderson 1997, Wegener 1996, Tokaty 1994, Vincenti 1990, Böhme 1978, Kármán 1954.)

Practical engineering problems in fluid dynamics - like flow of objects in water or air - have to take into account the inner friction of fluids or gases, i.e. their viscosity. The mathematical means that were drawn from the mathematical tradition in order to tackle these problems are the Navier-Stokes equations. (Major contributions to them were given by Navier in 1823, Cauchy in 1828, Poisson in 1829, Saint Venan in 1843 and Stokes in 1845.) These equations are a system of non-linear, high-order partial differential equations which pose formidable problems and do not have a general analytical solution.

A certain simplification of these equations is achieved if one assumes incompressibility of flow, which is given for flow speeds of less than a third of the speed of sound, i.e. for almost all liquid and many gas flows in everyday and engineering experience. Yet this does not help very much to overcome the difficulties of the equations. Since the viscosity of the majority of engineering problems is small, i.e. their Reynolds-number is high, the natural thing to do was to make another idealising assumption and try to find a solution by neglecting viscosity. (The Reynolds-number $R e$ is defined as $\rho V D / \mu$ which is equal to $V D / v . V$ is the velocity, $D$ a characteristic geometric dimension such as body length, pipe diameter or chord of an airfoil, $\rho$ the mass density, $\mu$ the dynamic viscosity constant and $v$ the kinematic viscos- 
ity of the fluid.) This approach was all the more attractive since the motion of inviscid incompressible steady flow was well understood and yielded explicit solutions for some body shapes. The mathematics for this is given by the Euler equations, which can be obtained from the Navier-Stokes equations by neglecting the friction terms. (From the Euler equations one can obtain the Bernoulli equation, which gives solutions to many concrete flow problems.)

However, as d'Alembert had already observed in 1768, the Euler equations yield a drag of zero for a moving body in an infinite stream. (Drag is the force experienced in the direction opposite to the body's movement.) A body moving through air or water would cause no force - a result which is falsified by everyday experience, e.g. the experience of throwing a ball. So even for problems involving low viscosity, mathematics could not help. Engineers had to rely on rules of thumb and experiences obtained from experimental model-building. Around the turn of the 20th century, the British chemist and Nobel laureate Sir Cyril Norman Hinshelwood lamented that fluid dynamists were divided into hydraulic engineers who observed things that could not be explained and mathematicians who explained things that could not be observed. (See Gad-el-Hak 1998, 181)

The discrepancy between the engineering and mathematics tradition in fluid dynamics was resolved with one stroke in a short paper by Prandtl in 1904. In this work, Prandtl gave an outline of his so-called "boundary layer" [Grenzschicht] theory. (Prandtl 1905) The concept of the boundary layer made it possible to separate the problem of viscous flow into two interacting components: The first component is a thin layer near the surface of a body, in which the velocity decreases from a finite value $U$ (the velocity of the outer flow) to zero at the surface of the body itself. The layer thus fulfils the so-called "no-slip" condition, first proposed by Daniel Bernoulli in 1738, which all known (real) fluids and gases satisfy. The second component of the problem is the flow outside of the boundary layer, which Prandtl assumed to be inviscid and thus amenable to the Euler equations. The flow of a body in a fluid (e.g. a flying aeroplane) or the flow around an object (e.g. the flow of a river against a pier) can now be seen as the result of an interaction between the two components. The decisive move is to assign a substantial causal role to the boundary layer and to confine the viscous effect to it. Although it is very thin in liquids like water and air, the layer has a considerable effect on the free flow because of its strong velocity gradient. (The higher the Reynolds-number and the shorter the body in the flow direction or the faster the velocity of the outer flow, the thinner 
the boundary layer gets.) One can say that modern fluid dynamics arose from a fusion of ideal-flow theory with all its splendid mathematics and the boundary layer concept. ${ }^{1}$

Prandtl's model made it possible for the first time to obtain approximate mathematical solutions for viscous flow. In the case of laminar stationary flow, the momentum and energy equations are parabolic and can therefore be calculated much more easily than in the NavierStokes case. The number of equations and unknown variables is thus reduced for the viscous part and the applicability of the Euler equations maintained for the outer flow. Prandtl thereby arrived at the following basic differential equations for stationary flow in two dimensions:

$$
\begin{array}{ll}
\rho(u \partial u / \partial x+v \partial u / \partial y)+d p / d x=k \cdot \partial^{2} u / \partial y^{2} \\
\partial u / \partial x+\partial v / \partial y=0 & \\
\text { Boundary conditions: } & \begin{array}{l}
\text { for } y=0: u=0, v=0 \\
\text { for } y=\infty: \mathrm{u}=U(x)
\end{array}
\end{array}
$$

$u$ and $v$ are the velocity components in $x$ and $y$ direction respectively, $\rho$ the density of the fluid, $p$ its pressure, $k$ its friction (viscosity constant; nowadays written as $\mu$ ). If $d p / d x$ and the development of $u$ in the layer are known, there exists a simple numerical approximation procedure to calculate the solution. Prandtl showed in his paper how to use a standard numerical technique to calculate the drag of water flow along a flat thin plate as caused by friction on the surface of a body. In 1907, Prandtl's student Heinrich Blasius was able to extend this to cylinders. (Blasius 1908) Since many engineering problems require the reduction of drag, e.g. on planes and ships, Prandtl's approach became all the more attractive.

In his paper, Prandtl also explained the phenomenon of flow separation and transition to turbulence in a qualitative way. As a result of surface friction, the boundary layer is put into rotation, separates from the surface and pushes its way through the outer flow. The separation marks a transition from a smooth, laminar state with low drag to a chaotic, turbulent one with higher drag. The problem of reducing drag is thus transformed into the problem of suppressing or delaying flow separation. For the first time, there was hope that the principles governing the problem may after all be found.

\footnotetext{
${ }^{1}$ The old separation of hydraulics and rational mathematics survived in a subtle but effective controversy between a purely mathematical approach to fluid dynamics and an applied one, as exemplified by the disciplinary confrontation between G. Birkhoff and Th. von Kármán in the 1940s and 50s. Birkhoff did not see the original sin of earlier mathematical fluid dynamics in
} 
Prandtl also presented experimental, albeit only qualitative, evidence for his proposal. He put a cylinder into a flow of water in which mica was suspended in order to visualise vortices and other deformations. The cylinder had a small slit exactly at the point where flow separation occurred. The fact that flow separation disappeared when water was pumped out of the cylinder and the boundary layer thus sucked off was proof for the hypothesis that there is in fact a boundary layer and that its interaction with the outer flow is responsible for the observed phenomena. (If the water had not been pumped out, separation of the flow from the cylinder wall would have occurred.)

In 1921, Prandtl's student Karl Pohlhausen showed in his dissertation that in many cases of flow the velocity distribution can be represented by relatively simple polynomials. He used the so-called "integral form" of the boundary layer equations as introduced by Theodore von Kármán the same year. This and similar developments made the boundary layer theory more and more attractive to working engineers. International reception of Prandtl's approach, however, was slow. It began to come in the 1930s and was not complete until after World War II.

In the early 1950s, S. Kaplun, P. A. Lagerstrom and M. Van Dyke developed a powerful mathematical technique for deriving asymptotic expansions of Navier-Stokes solutions. This so-called "method of matched asymptotics," which built upon results attained by K. O. Friedrichs and Prandtl in the 1940s, assumes that inner and outer solutions of the flow are connected - that is, that in their domains they share an overlapping region in which the solutions are equal.

It should be mentioned that Prandtl's boundary layer concept also proved fruitful for the study of heat transfer. Ernst R. G. Eckert hit upon this idea in the 1930s and summed it up in his Einführung in den Wärme- und Stoffaustausch of 1949. Eckert was among the 260 scientists and engineers invited by the Army Air Forces to come to the United States under "Operation Paperclip" in 1945 as was Karl Pohlhausen.

\section{The role of Prandtl's physical model}

What exactly is the purpose of Prandtl's model and how does it serve this purpose? As I have already pointed out the purpose of Prandtl's model is to make the partial differential equations applicable to cases that are interesting from an engineering point of view. The other goals,

the neglect of viscosity, as did Kármán, but in the lack of "deductive rigor" among engineers. (See Vincenti \& Bloor 2003) 
which go hand in hand with the first, are to broaden or deepen understanding of the physical phenomena and finally to make new testable predictions about them.

The important question to be raised now from a philosophical point of view is how these goals are achieved in Prandtl's model. Before I try an answer to this, I would like to invoke the distinction, proposed by Heinrich Hertz in the 1880 s and 1890 s, between a "theory" and its underlying "physical representation" [physische Vorstellung] (See Heidelberger 1998, 18). By "representation", Hertz meant the picture or physical idea of the intended objects of the theory. He also used the expressions "physical interpretation", "physical meaning", "images in thought" and "intuition" as alternatives to "representation." The form we give our representations, Hertz writes, "is such that the necessary consequents of the images in thought are always the images of the necessary consequents in nature of the things pictured." (Hertz 1894, 1)

From the "representation" of a theory, Hertz also distinguishes the theory's "presentation" or "expression" [Darstellung], i.e. the mathematical devices and concrete visual aids used to present it. Hertz had developed these distinctions in dealing with Maxwell's electrodynamics and its contemporary rivals. He took into account that a theory of a certain domain can be compatible with different physical representations. A follower of Helmholtz's representation of electrodynamics in terms of potentials and action at a distance could accept Maxwell's equations without being forced to accept Maxwell's mature representation of them in terms of electromagnetic fields. "The representation of the theory in Maxwell's own work, its representation as a limiting case of Helmholtz's theory, and its representation in the present dissertations [i.e. his own representation] have substantially the same inner significance [= mathematical content].” (Hertz 1892, 21)

Can we take Hertz's conception over into fluid dynamics and use Hertz's concept of representation to characterise Prandtl's physical model? Not without a certain modification. Prandtl's model is not a physical representation of the Navier-Stokes equations, but of a theory whose mathematical content is an approximation to these equations. In a more general way, then, we can say that a physical model of a set of continuity equations is either a physical representation of these equations themselves or of a mathematical structure that approximates the equations.

In Prandtl's case, this would amount to the following: The fundamental theory, as presented by the Navier-Stokes equations, does not suggest by itself how it is to be represented physically. Such a representation is added through Prandtl's assumption that object flow has to be conceived as the interaction of viscous and inviscid flow. This model is consistent and 
experimentally confirmed, but it is not a representation of the Navier-Stokes equations as such. Instead, it represents an approximation to the Navier-Stokes equations that is applicable to practical engineering problems.

Using this terminology, we can rephrase the question of exactly how Prandtl achieves his goals, breaking it up into two parts: 1. How does Prandtl's model differ either from received physical representations of the basic equations or from representations of approximations to them? 2. How does it affect the theory and its presentation?

Michael Redhead has introduced a distinction between two kinds of models in physics: those that are "enriched" theories and those that are "impoverished" ones. (Redhead 1980) Sergio Sismondo has pointed out that this dichotomy cannot be exclusive since a model can be an enriched and impoverished theory at the same time. (Sismondo 1999, 253f.) Instead of saying that a model further develops a theory by enriching or impoverishing its assumptions, I prefer to say, in accordance with Hertz, that a model is not a theory in itself but a representation of a theory. Thus, a new model of a theory modifies a received representation of a theory by enriching or impoverishing it.

This suggestion can be spelled out for the Prandtl case in the following way: The physical representation normally accompanying the Navier-Stokes equations is enriched by the concept of the boundary layer. (This answers the first question.) The enrichment leads to a simplification (impoverishment) of the theory itself (or, if you wish, of its mathematical presentation) by substituting an approximation for it that changes the elliptic character of the Navier-Stokes equations into a much more easily handled parabolic character. (This is part of the answer to the second question.) It must be stressed that enrichment cannot be seen as the mere addition of a new factor to an otherwise unaffected theory, nor impoverishment as the mere neglect or elimination of useless theory elements, without which the core of the theory remains intact. Nor can these two processes be seen as correcting a theory by substituting true elements for wrong ones. A much more apt description of this modelling process would be to use Thomas Kuhn's expression and call it "theory articulation." In Winsberg's words this is the "nontrivial process of bringing a theoretical structure into resonance with some phenomena." (Winsberg 1999, 286) In contrast to Kuhn, however, and in accordance with the Prandtl case, this process cannot be conceived just as the spelling out of what is already implicit in the paradigm. I wholeheartedly agree with Winsberg that in mature sciences theory articulation is a form of genuinely new knowledge production.

As a consequence, applying a theory to concrete engineering situations, i.e. making it deliver a description of certain processes in its domain, is not just a matter of bringing a de- 
scriptive content to the fore that is already inherent and implicit in the theory. Rather, it is a creative process whereby a model of an approximation to the theory is found that makes it possible to solve a problem which otherwise would remain unsolved. This makes Prandtl's move a nontrivial enterprise.

Making the mathematics tractable is one effect (and one purpose) of Prandtl's model. It also plays the role of broadening and deepening our understanding of fluid flow. What this means should be spelled out in more detail. Carl G. Hempel explicitly proposed the view that broadening and deepening understanding has to be conceived of as an improvement of a covering law explanation. A new explanation achieves an increase in breadth in relation to a precursor if the theory covers "a wider range of occurrences than do the empirical laws previously established." And it deepens our understanding if at least one of the following conditions is fulfilled: 1. "It reveals the different regularities exhibited by a variety of phenomena $[\ldots]$ as manifestations of a few basic laws." 2. It describes the empirical generalisations known so far as good approximations of a new fundamental theory within a certain limited range. (Hempel 1965, 345) One gets the impression that in developing this idea Hempel generalised the standard account of the Kepler-Newton relationship, on the one hand, and the NewtonEinstein relation on the other. Newton's laws encompass Kepler's laws and many other previously unconnected ones (= broadening of understanding), and Newton's laws approximate the solutions of special relativity theory for small velocities, i.e. within a limited range (= deepening of understanding).

On this account, Prandtl's model cannot be described as providing a more profound understanding of flow than its predecessors. The new regularities of the boundary layer have not been deduced from the Navier-Stokes equations. And, although considering the boundary layer as a limiting case might have played a role in Prandtl's mind, neither has the model been deduced from the Navier-Stokes equations as an approximate case within a certain limited range. A claim like this would not do justice to the ingenuity with which Prandtl resolved d'Alembert's paradox. Yet, there is some truth in Hempel's suggestion. He was right that a theory provides a better explanation when it increases the coherence of a domain, but he was wrong when he thought that this is only achieved through laws.

There is also an alternative view according to which a phenomenon is understood by invoking or identifying a mechanism that produces it (Machamer, Darden, Craver 2000; Cushing 1998, 338-342) or by showing how it fits into a pattern of causal processes and their interactions. (Salmon 1984) Would it help to reject the covering law account and adopt the mechanism view of scientific understanding? This would certainly bring out a feature of 
Prandtl's model that is neglected by the covering law account. The key idea is indeed to account for the phenomena in terms of a hitherto unidentified causal process, namely the interaction between the boundary layer and the outer flow. Prandtl decomposed the flow process into separate localised causal components.

Yet this achievement would improve understanding only to a very limited degree if it were not backed up by the simplification of the Navier-Stokes equations, i.e. if it did not give us a hint as to how the parameters concerned are quantitatively related to each other. This "unificatory" aspect of Prandtl's model is not automatically supplied by detailing a causal mechanism. So it is true that the mechanism-view is better than the covering law account in judging Prandtl's model, but it gives only half the story.

There is another alternative available for explicating what it means to understand a phenomenon. This is the semantic conception of theory, which identifies theory itself with a population of models that are homomorphically related to systems in the real world. At first, this might seem attractive for Prandtl's case, since it allows us to see the success of his theory in the descriptive adequacy of its basic model. Improving our understanding of a phenomenon would thus mean giving a model of it which has more features in common with the system in the real world than earlier accounts. A deeper look reveals, however, that descriptive adequacy cannot be enough. It is also relevant how this adequacy is achieved, indeed crucially so. If a model were made to fit a phenomenon by an ad hoc move, we would reject it even if it were descriptively more adequate than an alternative. So, in the end, the semantic conception does not tell us how Prandtl's model achieves scientific understanding. Consequently, the semantic conception relegates the question of how a theory achieves explanatory success to the cognitive sciences, which would have to decide by empirical research into the behaviour of scientists whether a theory is explanatory or not. As Giere writes, the question "is not to be judged by philosophical standards." (Giere 1988, 105) So again, only half the story is told: According to the semantic view, Prandtl's model gives a better description of the phenomena because it provides a more effective way of dealing with the differential equations, but this view overlooks the causal character of the model.

What exactly is it, then, that is responsible for deepening our understanding in the case of Prandtl? The answer to this question must lie somewhere in between the covering law account and the mechanism conception. Prandtl's model introduces a coherence that previously could not be attained without appealing to overarching laws, and it provides a causal story into which the phenomena can be fitted without forgetting about unification. Prandtl's model has a lot in common with what Thomas Kuhn called an "exemplar:" It provides a "concrete 
problem-solution" that makes it possible to view other cases of application as analogous to the original problem. "Scientists solve puzzles by modeling them on previous puzzle-solutions, often with only minimal recourse to symbolic generalizations.” (Kuhn 1970, 189f.)

It is interesting to see how contemporaries received Prandtl's work. It seems that they especially valued the coherence and fruitfulness of the new approach. By "coherence" I mean the property of making few or no ad hoc assumptions. Hence, Blasius, whom I mentioned earlier, stressed the greater coherence of Prandtl's theory in comparison with the theory of his predecessor Hermann von Helmholtz. The major advance is seen in the coherent explanation of flow separation: It is true that Helmholtz explained this phenomenon by juxtaposing two potential (i.e. inviscid and rotation-free) flows, namely a moving and a stationary one, and by assuming that pressure decreases to zero at their point of contact, but his theory is not able to take viscosity into account. In particular, it does not consider the no-slip condition and therefore cannot explain the origin of vortices after the separation point. In addition, there is no zero pressure at the meeting point of the two flows assumed. In contrast, Prandtl's explanation, Blasius writes, “does not operate with an ad hoc assumption as Helmholtz's theory of rays does, but only with the representations [Vorstellungen] which form the basis of our hydrodynamic equations.” (Blasius 1908, 4). In a similar way, Prandtl had stressed in his first contribution that "the most important result of these [= his] investigations for application" is the explanation of flow separation. (Prandtl 1904, 578) By overcoming the ad hoc character of Helmholtz's theory, Prandtl's theory promised analogous solutions to other cases of application that puzzled his contemporaries.

Is Prandtl's search for coherence a search for unification? Is the understanding brought about by Prandtl's model due to its explanatory unity? If unity were taken to mean sparsity of the laws used (as is the case with the main advocates of the unification view, Friedman 1974 and Kitcher 1989) then the answer to these questions would have to be "no." If only the economical efficiency of the law were taken into account, the Navier-Stokes equations would be more unificatory than Prandtl's model. Yet, if unification is taken to mean a close relationship among the elements used or a high structural coherence among them, then "unification" would indeed be the right expression to characterise Prandtl's advance over the rational mathematicians and especially over his predecessor Helmholtz. Prandtl does not achieve this unification through one or more laws but through a physical model which yields the desired understanding with one stroke. 


\section{Computational and experimental models in fluid dynamics}

In computational fluid dynamics, computers are used to numerically analyse problems in order to simulate or predict fluid flow. Usually, the flow is decomposed ("discretised") into small discrete cells that form a grid ("the mesh") to which iterative methods are applied. The discretisation of the Navier-Stokes equations (which are continuum equations) can be accomplished in many ways: by means of finite difference, finite-volume, finite-element, or spectral methods etc. Each of these strategies has its own philosophy and programming style for obtaining a set of discrete algebraic equations. By using an appropriate numerical algorithm, the evolution of the flow-field is computed by solving the discrete equations for well-defined initial and boundary conditions. This is done under certain idealising assumptions in order to keep the parameters manageable. Very often, the solution given by the computer is visualised so that flow characteristics can readily be grasped. The process of computational modelling comprises three phases: a physical modelling phase, a numerical approximation phase and a mapping phase. In a relatively new modelling strategy, the so-called Lattice-Boltzmann models, based on particles and cellular automata principles, the first and second phases are combined.

There are several reasons for the growing use of computational models. They give approximate solutions to the Navier-Stokes equations; reduce the time span for parameter variation, design and development of devices; are cheaper than experimental modelling; and can simulate flow conditions that are not accessible to experimental testing. Sources of error for computational models can lie in numerical instabilities whereby small errors at each step accumulate, in the low accuracy of the model, or in its imperfect verification or validation. "Verification" is taken to mean the testing of the model in relation to existing analytical solutions or against a known computational solution that can serve as a benchmark, encapsulating the physics of the actual problems to be solved in as many aspects as possible. "Validation," on the other hand, is confirmation by experimental means. Since every computational model must incorporate some idealisation in order to be efficient, this is no easy task.

Turbulent flow is a special case in all this. It cannot be approached analytically. In order to make up for this, special terms have to be introduced that are often based on purely empirical knowledge. Insight into turbulence seems to be confined so far to experimenting (by physical or numerical means). Because of its high expenditure, direct numerical simulation (DNS) (simulation for vanishing cells, without averaging) has only been achieved up to a Reynolds-number of 1410. A supercomputer with $10^{18}$ floating-point operations would be 
needed to simulate a complete aeroplane via DNS. (Gad-el-Hak 1998, 182f.) Presently, $10^{12}$ flops have yet to be exceeded.

After this overview of computational modelling in fluid dynamics, we may address the question of how computational models differ from physical ones and the question as to what the term "model" means when talking of computational models. Remember that we characterised a physical model as a physical representation of a theory whose mathematical content is an approximation to (part of) the basic continuity equations of the theory. The most natural definition that comes to mind would be the following: a computational model of a theory is the mathematical representation of the decomposition of the continuous domain into discrete elements and its implementation in producing approximate numerical solutions.

Although computational modelling has greatly reduced the need for experimental modelling, the latter is still indispensable in fluid dynamics. We have already pointed out that mastering turbulence necessarily demands the use of empirical elements. But even the most powerful computer program has to be validated empirically at some point or other, direct or indirectly, and the boundary conditions must be determined. Indeed, the demand for greater exactness of experimental methods to match the high resolution power of computational models has increased drastically. This demand has been met by sophisticated new measuring devices, such as non-invasive flow velocity meters with high temporal resolution, which are based on the Doppler effect and implement laser technology, and miniature devices for the measuring of pressure.

Very recently, experimental observation - or, rather, its interplay with theory and mathematics - has enjoyed unexpected triumph and promises a new approach to solving the hard problem of fluid dynamics, namely turbulence. On the basis of computational studies and ideas drawn from dynamical systems theory, a model for the transition process to turbulence in pipe flow has been developed. It is based on the conception of unstable travelling waves. These waves have been observed with surprising clarity and seen to agree with numerical studies. (Hof et al. 2004, Busse 2004) This breakthrough nourishes the hope that a full understanding of the transition problem is possible after all and that theoretical fluid dynamics will be able to influence and control turbulence transitions in the not too distant future.

To round off our discussion of models, we should reflect a bit more on experimental models. Can the use of the term "model" in this context be adjusted to match the use of the terms "physical model" and "computational model" that I have suggested? I think it can. An experimental model is the concrete material representation of the physical model. The representation is to be achieved in such a way that it enables the manipulation and control of the 
parameters. Think of Prandtl sucking away the boundary layer: The experimental set-up makes use of the insight provided by the physical model to decrease drag.

\section{Conclusion}

It should be obvious by now that applying a theory in fluid dynamics is not just a matter of deriving a special case from a general theory under boundary conditions. Rather, it means finding a physical model that can lead to an approximate solution of the fundamental continuity equations. As I have characterised the three model types in fluid dynamics, the physical model is attributed a central position. Both the computational and the experimental model have to rely on it at some point in order to do their work properly, even though their contact with the physical model may be rare or inconspicuous.

In this context, the question arises how the representational function usually accorded to a theory is distributed between the general theory and the physical model. There are two possibilities for interpreting the situation: One could say that the Navier-Stokes equations describe reality, but that we do not have direct access to this description for individual concrete cases, and therefore must be content with an approximation given in a physical model. This view makes the model the handmaiden of the theory and stresses its mediating position between theory and experience. On the other hand, however, we would be equally right to say that the differential equations are just part of a formal technique for letting physical models speak quantitatively. In this case, the physical model is seen as an autonomous agent that calls the continuity equations to its service and uses them in a purely instrumentalist way. In short, the dichotomy is a bit like that between Plato's ante rem realism regarding universals and Aristotle's in re realism as it arises in the philosophy of mathematics.

Fortunately, we do not have to solve a problem from the philosophy of mathematics here. For engineering science, we should decide this question in the following way. We should look in which direction realism tends, i.e., we should find out how the inferences to the best explanation are drawn in the practice of engineers and scientists: Do their conclusions deal with the existence of the theoretical entities postulated by the theory or with the existence of those postulated by the model? I think that, at least in Prandtl's case, the scale definitely tips to the Aristotelian side, i.e. the side of the models. Prandtl argues first for the existence of the boundary layer and the existence of a perturbation process responsible for the separation of the flow. Only after confidence in this result has been established is it meaningful to ask how it can be formulated in and be adjusted to the frame of the continuity equations by systematically neglecting certain parameters in them. So Prandtl's model is not a fictional or ide- 
alised tool that enables the theory to be applied. It is the other way around: The model tells us something about the world, whereas the fundamental theory (the Navier-Stokes equations) is so abstract and malleable that it does not deliver any definite information about the concrete case, i.e. it does not even lie about it.

\section{References}

Anderson, J. D. (1997). A History of Aerodynamics and Its Impact on Flying Machines. Cambridge: Cambridge University Press.

Blasius, H. (1908). "Grenzschichten in Flüssigkeiten mit kleiner Reibung.” Zeitschrift für Mathematik und Physik 56(1): 1-37.

Böhme, G. (1978). “Autonomisierung und Finalisierung.” Starnberger Studien, vol. 1: Die gesellschaftliche Orientierung des wissenschaftlichen Fortschritts. Ed. G. Böhme et al. Frankfurt am Main: Suhrkamp, 69-118 (pp. 81-113 on fluid mechanics).

Busse, F. H. (2004). "Visualizing the Dynamics of the Onset of Turbulence." Science 305: 1574-1575.

Cartwright, N. (1983). How the Laws of Physics Lie. Oxford: Clarendon.

Cushing, J. T. (1998). Philosophical Concepts in Physics: The Historical Relation between Philosophy and Scientific Theories. Cambridge: Cambridge University Press.

Eckert, E. R. G. (1949). Einführung in den Wärme- und Stoffaustausch. Berlin/ Göttingen/ Heidelberg: Springer.

Friedman, M. (1974). "Explanation and Scientific Understanding.” Journal of Philosophy 71: 5-19.

Gad-el-Hak, M. (1998). "Fluid Mechanics from the Beginning to the Third Millenium.” International Journal of Engineering Education 14(3): 177-185.

Germain, P. (2000). “The 'NEW' Mechanics of Fluids of Ludwig Prandtl.” In Meier (2000), 31-40.

Gersten, K. (2000). "Ludwig Prandtl und die asymptotische Theorie für Strömungen bei hohen Reynolds-Zahlen.” In Meier (2000), 125-138.

Giere, R. N. (1988). Explaining Science: A Cognitive Approach. Chicago: University of Chicago Press. (Repr. 1990)

Graebel, W. P. (2001). Engineering Fluid Mechanics. New York/ London: Taylor \& Francis.

Heidelberger, M. and F. Steinle, eds. (1998). Experimental Essays - Versuche zum Experiment. (ZiF - Interdisciplinary Studies. Vol 3.) Baden-Baden: Nomos. 
Heidelberger, M. (1998). From Helmholtz's Philosophy of Science to Hertz's Picture-Theory. In Heinrich Hertz: Classical Physicist, Modern Philosopher. Ed. Davis Baird, R. I. G. Hughes and Alfred Nordmann. Dordrecht: Kluwer, 9-24.

Hempel, C. G. (1965). Aspects of Scientific Explanation. New York: Free Press.

Hertz, H. (1892). Electric Waves being Researches on the Propagation of Electric Action with Finite Velocity Through Space. London/ New York: Macmillan 1893. Repr. New York: Dover 1962. (Orig. Untersuchungen über die Ausbreitung der elektrischen Kraft. Leipzig: Barth 1892.)

Hertz, H. (1894). The Principles of Mechanics Presented in a New Form. London/ New York: Macmillan 1899. Repr. Mineola, NY: Dover 2003. (Orig. Gesammelte Werke, Bd. III: Die Prinzipien der Mechanik in neuem Zusammenhange dargestellt. Leipzig: Barth 1894.)

Hof, B. et al. (2004). "Experimental Observation of Nonlinear Traveling Waves in Turbulent Pipe Flow." Science 305: 1594-1598.

Jacob, K. (2000). “A Review of Ludwig Prandtl's Scientific Life.” In Meier (2000), 17-29.

Kármán, T. v. (1954). Aerodynamics: Selected Topics in the Light of Their Historical Development. Ithaca, NY: Cornell University Press.

Kitcher, P. (1981). “Explanatory Unification.” Philosophy of Science 48: 507-531.

Kuhn, T. S. (1970). The Structure of Scientific Revolutions. (2nd enlarged ed.) Chicago: The University of Chicago Press.

Machamer, P., L. Darden and C. F. Craver (2000). "Thinking About Mechanisms.” Philosophy of Science 67: 1-25.

Meier, G. E. A. ed. (2000). Ludwig Prandtl, ein Führer in der Strömungslehre. Biographische Artikel zum Werk Ludwig Prandtls. Braunschweig: Vieweg.

Morgan, M. S. and M. Morrison, eds. (1999). Models as Mediators: Perspectives on Natural and Social Science. Cambridge: Cambridge University Press.

Morrison, M. (1999). “Models as Autonomous Agents.” In Morgan/ Morrison (1999), 38-65.

Pohlhausen, K. (1921). "Zur näherungsweisen Integration der Differentialgleichung der laminaren Grenzschicht.” Zeitschrift für angewandte Mathematik und Mechanik 1: 252-268.

Prandtl, L. (1905). "Über Flüssigkeitsbewegungen bei sehr kleiner Reibung.” Verhandlungen des 3. internationalen Mathematiker-Kongresses in Heidelberg vom 8. bis 13. August 1904. Ed. A. Krazer. Leipzig: Teubner: 484-491. (Repr. in Prandtl (1961) 2: 575-584).

Prandtl, L. (1931). Abriß der Strömungslehre. Braunschweig: Vieweg.

Prandtl, L. (1948). "Mein Weg zu hydrodynamischen Theorien.” Physikalische Blätter 4(3): 89-92. (Repr. in Prandtl (1961) 3: 1604-1608.) 
Prandtl, L. (1961). Gesammelte Abhandlungen zur angewandten Mechanik, Hydro- und Aerodynamik. 3 vols. Berlin/ Göttingen/ Heidelberg: Springer.

Redhead, M. (1980). "Models in Physics.” British Journal for the Philosophy of Science 31: 481-520.

Rouse, H. and S. Ince (1963). History of Hydraulics. New York: Dover.

Salmon, W. C. (1984). Scientific Explanation and the Causal Structure of the World. Princeton: Princeton University Press.

Siekmann, H. (1998). "Experiment und Simulation in der Strömungstechnik.” In Heidelberger/ Steinle (1998), 209-226.

Sismondo, S. (1999). "Models, Simulations, and Their Objects.” Science in Context 12(2): 247-260.

Smits, A. J. (1999). A Physical Introduction to Fluid Mechanics. New York: Wiley.

Ting, L. (2000). "Boundary Layer Theory to Matched Asymptotics." Zeitschrift für angewandte Mathematik und Mechanik 80(11-12): 845-855.

Tokaty, G. A. (1994). A History and Philosophy of Fluid Mechanics. New York: Dover. (1st ed. 1971)

Vincenti, W. G. (1990). What Engineers Know and How They Know It: Analytical Studies from Aeronautical History. Baltimore/ London: The Johns Hopkins University Press.

Vincenti, W. G. a. D. B. (2003). "Boundaries, Contingencies and Rigor: Thoughts on Mathematics Prompted by a Case Study in Transonic Aerodynamics.” Social Studies of Science 33(4): 469-507.

Wegener, P. P. (1996). What Makes Airplanes Fly? History, Science, and Applications of Aerdodynamics. New York/ Berlin/ Heidelberg: Springer. (1st ed. 1991)

Winsberg, E. (1999). "Sanctioning Models: The Epistemology of Simulation." Context 12(2): 275-292.

Winsberg, E. (2003). "Simulated Experiments: Methodology for a Virtual World." Philosophy of Science 70: 105-125.

Zierep, J. (2000). “Ludwig Prandtl, Leben und Wirken.” In Meier (2000), 1-16. 